\title{
Construction of asymmetric copulas and its application in two-dimensional reliability modelling
}

\author{
Shaomin $\mathrm{Wu}^{1}$ \\ Kent Business School, University of Kent, \\ Canterbury, Kent CT2 7PE, United Kingdom
}

\begin{abstract}
Copulas offer a useful tool in modelling the dependence among random variables. In the literature, most of the existing copulas are symmetric while data collected from the real world may exhibit asymmetric nature. This necessitates developing asymmetric copulas that can model such data. In the meantime, existing methods of modelling two-dimensional reliability data are not able to capture the tail dependence that exists between the pair of age and usage, which are the two dimensions designated to describe product life. This paper proposes a new method of constructing asymmetric copulas, discusses the properties of the new copulas, and applies the method to fit two-dimensional reliability data that are collected from the real world. Keywords: copula, tail dependence, warranty, two-dimensional reliability data, asymmetric.
\end{abstract}

\section{Introduction}

\subsection{Motivation}

Copulas are a tool for constructing multivariate distributions and formalising the dependence structures between random variables. The notion of copula was first introduced by Abe Sklar in 1959 when he responded to a question with respect to the relationship between a multidimensional

1 Email: s.m.wu@kent.ac.uk. Telephone: 00441227827940.

Suggested citation: S. Wu, Construction of asymmetric copulas and its application in twodimensional reliability modelling, European Journal of Operational Research, 238(2), pp. 476-485. DOI: 10.1016/j.ejor.2014.03.016. 
probability function and its lower dimensional margins [1]. It has attracted considerable attention in recent years in both theoretical and application aspects.

Sklar's Theorem states that any cumulative distribution function of a random vector can be written in terms of marginal distribution functions and a copula that describes the dependence structure between the variables [1]. That is, given a vector of random variables $\left(X_{1}, \ldots, X_{d}\right)$, its cumulative distribution function $H\left(x_{1}, \ldots, x_{d}\right)\left(=P\left(X_{1} \leq x_{1}, \ldots, X_{d} \leq x_{d}\right)\right)$, and marginals $F_{k}\left(x_{k}\right)\left(=P\left(X_{k} \leq x_{k}\right)\right.$, where $\left.k=1, \ldots d\right)$, Sklar proved that $H\left(x_{1}, \ldots, x_{d}\right)$ can be written as $H\left(x_{1}, \ldots, x_{d}\right)=C\left(F_{1}\left(x_{1}\right), \ldots, F_{d}\left(x_{d}\right)\right)$ and named $C($.$) as a copula [1]. Copulas are useful in sta-$ tistical applications because they allow one to easily model and estimate the distribution of a random vector through estimating the marginals and the copula separately.

In the literature, many parametric copula families have been proposed $[2,3]$. Most of them are symmetric in the sense that the variables in a copula are exchangeable, $C\left(v_{1}, v_{2}\right)=C\left(v_{2}, v_{1}\right)$ $\left(v_{1}, v_{2} \in[0,1]\right)$ for the bivariate copula case, for example. However, there are many natural processes that possess asymmetric dependence structures. Using symmetric copulas to model such processes may not be able to capture the nature of the data. This necessitates developing asymmetric copulas. This paper serves this necessity by developing a method of constructing asymmetric copulas.

\subsection{Related work}

\subsubsection{Construction of asymmetric copulas}

There are many copula families. The reader is referred to the monographs by Joe [2] and Nelsen [3] for detailed accounts of the theory and surveys of commonly used copulas and to the review papers [4-6], work on tail dependence $[7,8]$, and papers on applications $[9,10]$.

Some work has been done for constructing asymmetric copulas ([11,12], for example). Alfonsi and Brigo [11] described a method based on periodic functions. Liebscher [12] introduced two methods to construct asymmetric multivariate copulas: the first is connected with products of copulas and the second generalises the Archimedean copulas, and the resulting copulas are asymmetric. However, their research did not show how those methods can be used to construct asymmetric copulas with tail dependence in a given direction.

\subsubsection{Modelling two-dimensional reliability data}

Estimating reliability functions is important for both product manufacturers and asset managers. System reliability is usually estimated based on one dimension, either usage (for example, the number of door openings of an elevator, the number of pages copied by a copy machine) or age. 
In reality, however, failures or other adverse events in systems may depend on both the age and the usage history of the systems. Such examples can be found in warranty claim data analysis, in which the warranty policy for certain types of products specifies the limits of coverage in terms of both age and usage. For example, a sold car may be covered by a 3 year and 30,000 mile warranty, which implies that the warranty supplier - which is usually the manufacturer - will repair the car if it fails within the warranty coverage. It is important for the manufacturer to estimate the reliability of their products for the purpose of product improvement and fiscal planning. There is considerable research on warranty data analysis. For more detailed discussion, the reader is referred to recently published review papers $[13,14]$ and papers on reliability data analysis (see [15-17], for example).

There are three methods that have been developed for analysing two-dimensional reliability data. Those methods are briefed below.

The univariate method. It indirectly estimates $F\left(x_{1}, x_{2}\right)$ through the following two steps: to estimate $F_{2}\left(x_{2} \mid x_{1}\right)$ and $F_{1}\left(x_{1}\right)$, separately, then to obtain $F\left(x_{1}, x_{2}\right)=F_{2}\left(x_{2} \mid x_{1}\right) F_{1}\left(x_{1}\right)$ (where $x_{1}$ and $x_{2}$ represent age and usage, respectively). This method treats the usage as a function of the age $[18,19]$.

The bivariate method. It directly estimates $F\left(x_{1}, x_{2}\right)$ from data. For example, Jung and Bai [20] developed a bivariate method and assumed that the bivariate distribution can describe the positive correlation between the age and the usage.

The time scale method. In addition to the above two methods, Gertsbakh and Kordonsky [21] proposed a method that integrates the two scales (age and usage) to create a single composite scale and failures are modelled as a counting process. For example, in Gertsbakh and Kordonsky [21], a new variable $Z=\alpha X_{1}+(1-\alpha) X_{2}$ is introduced, where $\alpha \in(0,1), X_{1}$ and $X_{2}$ are the random variables representing the age and the usage, respectively.

However, the above methods have the following drawbacks.

- Both the univariate and the time scale methods assume a relationship, for example, a linear relationship, between the usage and the age, and then derive a bivariate joint lifetime distribution. A drawback of those two methods is apparent because such an assumption may be violated.

- Compared with the univariate method and the time scale method, the bivariate method is simpler and more straightforward, in the sense that the former two methods need two steps: first to estimate the relationship between age and usage and then estimate a joint distribution, but the bivariate method needs only one step that directly estimates a joint distribution. When the bivariate method is utilised, however, one needs to select a bivariate distribution in which the two variables are positively correlated. Furthermore, when the usage is measured as a discrete random variable (the number of pages copied by a copy machine, for example) and the age is described as a continuous random variable, to find a bivariate distribution that 


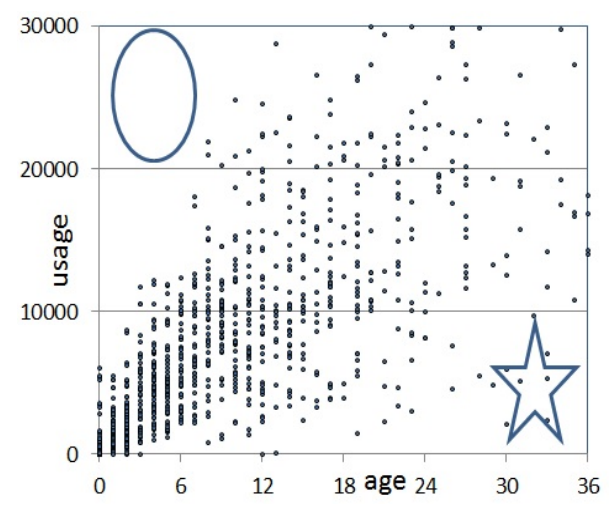

Fig. 1. Warranty claim data

combines a discrete marginal with a continuous marginal may be difficult.

- In addition to the above two drawbacks, an important fact has never been addressed in the existing literature so far. This fact is the existence of the tail dependence between age and usage, as explained below.

(a) If the age of the product is small, its usage should be small. This is because the age is the calendar time and it is not possible to develop large cumulative usage within a short period of the calendar time. Another reason is due to the operating limit, for example, a car usually cannot be driven faster than 100 miles per hour, hence the usage within a time interval is limited.

(b) If the age is large, on the other hand, the usage can be small. For example, some cars are not frequently used. Hence, although they are very old, their mileage can be very small.

Those two points (a) and (b) can also be observed from Figure 1, which is a scatterplot with the age on the $\mathrm{X}$-axis and the usage on the $\mathrm{Y}$-axis of the warranty claims of a particular model of car. The data are collected from a car manufacturer. As can be seen, there is no observation in the left-upper region in the figure (marked with the ellipse), which agrees with point (a). But there are some observations in the right-lower region in the figure (marked with the star), which agrees with point (b).

The above two points (a) and (b) can also be observed in those warranty data illustrated in Figure 2 of Alam and Suzuki [19] and in Figure 2 of Rai and Singh [22].

\subsection{Contribution and importance of this work}

This paper proposes a new method to construct asymmetric copulas and then applies the method to model two-dimensional reliability data. Its contribution is summarised in the following.

- It proposes a new method of constructing asymmetric copulas and a convex-combination of asymmetric copulas that can exhibit different tail dependence along different directions.

- It is the first paper to use asymmetric copulas to model the lifetime distribution on two- 
dimensional reliability data.

The proposed method has the following merits.

Ability to handle tail dependence. The proposed method of construction of asymmetric copulas differs from other existing methods in the sense that it can directly construct a copula with tail dependence in a given direction.

No limitation on the form of the marginal distributions. With copulas, one can select whichever two distributions to combine with in modelling reliability data, which can overcome the difficulty of selection of bivariate distributions, this is especially true for the case when the variables (age and usage, for example) come from different parametric families (one of a discrete random variables and one of a continuous random variable, for example).

The paper has important managerial implications. Derivation of the lifetime distribution for systems is important for both product manufacturers and users $[13,14]$.

- For the product manufacturers, product warranty has become increasingly more important in consumer and commercial transactions and is widely used to serve many different purposes as the US Congress has enacted several acts (UCC, Magnusson Moss Act, Tread Act, etc.) over the last 100 years and the European Union (EU) passed legislation requiring a two-year warranty for all products sold in Europe. The proposed method to estimate the lifetime distribution for the products under two-dimensional warranty can be beneficial to the manufacturers in estimating product reliability and forecasting future warranty claims needed for preparing warranty reserves plans.

- For product users, the proposed method can be used in their life cycle performance analysis or asset management.

Although this paper is developed using examples borrowed from reliability theory, its results and discussion can also be applied to any situations when random variables exhibit an asymmetric relationship. Such applications can be found in other disciplines. For example, the proposed method can be used to model the flow velocity and the wind speed [23].

\subsection{Overview}

The remainder of the paper is structured as follows. Section 2 introduces the concept of copulas, dependence measures, and asymmetric copulas. More importantly, it introduces a method of constructing asymmetric copulas, studies the properties of the constructed copulas, and then applies the method of constructing asymmetric copulas to the bivariate copula case. Section 3 discusses the existing methods of modelling two-dimensional reliability data, proposes two required conditions for modelling two-dimensional warranty data, and then compares the performance of the proposed method to two existing methods based on warranty data collected from a car 
manufacturer. Section 4 concludes the findings of this work and suggests our future work.

\section{Two methods of constructing asymmetric copulas}

In this section, we introduce the concept of the copula and tail dependence coefficient, and study the properties of the proposed copulas.

\subsection{Introduction to copulas}

Assume a $d$-dimensional random vector $\mathbf{X}\left(=\left(X_{1}, \ldots, X_{d}\right)\right)$ with marginal cumulative distribution functions $F_{1}\left(X_{1}\right), \ldots$, and $F_{d}\left(X_{d}\right)$. Sklar's theorem then proved that the joint distribution $F\left(X_{1}, \ldots, X_{d}\right)$ of the random vector $\mathbf{X}$ can be written as a function of its marginal distributions, ie., $F\left(X_{1}, \ldots, X_{d}\right)=C\left(F_{1}\left(X_{1}\right), \ldots, F_{d}\left(X_{d}\right)\right)$ [1]. The function $C($.$) is called a copula, which can$ also be defined as following.

Definition 1 (Copula) Denote $[0,1]$ by $\mathbf{I}$. A copula of dimension $d$ is a function $C: \mathbf{I}^{d} \rightarrow \mathbf{I}$ with the following properties:

(a) (Grounded) $C\left(v_{1}, \ldots, v_{d}\right)=0$, if $\exists k \in\{1, \ldots, d\}$ with $v_{k}=0$,

(b) (Consistence with margins) $C\left(1, \ldots, 1, v_{k}, 1, \ldots, 1\right)=v_{k}$, for $\forall v_{k} \in \mathbf{I}$ and $k \in\{1, \ldots, d\}$,

(c) (d-increasing) $\sum_{k_{1}=1}^{2} \ldots \sum_{k_{d}=1}^{2}(-1)^{k_{1}+\ldots+k_{d}} C\left(v_{1, k_{1}}, \ldots, v_{d, k_{d}}\right) \geq 0$, for $\forall v_{k, 1}, v_{k, 2} \in \mathbf{I}$ and $k \in\{1, \ldots, d\}$.

Property (a) reflects the fact that the joint probability of all outcomes is zero if the marginal probability of any outcome is zero. Property (b) tells that if the realizations of any $d-1$ variables have marginal probability ones, then the joint probability of the $d$ outcomes is the probability of the remaining uncertain outcome. Property (c) shows the non-decreasing nature of the cumulative probability distribution.

Many copulas have been proposed. The reader is referred to monographs $[2,3]$ for more comprehensive introduction. For example, below are two popular copulas.

- The Clayton copula [3]. The Clayton copula is given by

$$
C\left(v_{1}, \ldots, v_{d}\right)=\left(\sum_{k=1}^{d} v_{k}^{-\theta}-1\right)^{-1 / \theta}
$$

where $\theta \in[-1,0) \cup(0,+\infty)$. 
- The Gumbel copula [3]. The Gumbel copula is given by

$$
C\left(v_{1}, \ldots, v_{d}\right)=\exp \left\{-\left[\sum_{k=1}^{d}\left(-\ln v_{k}\right)^{\theta}\right]^{1 / \theta}\right\},
$$

where $\theta \in(0,+\infty)$.

\subsection{Tail dependence}

In essence, a copula $C\left(v_{1}, \ldots, v_{d}\right)\left(=P\left(F_{1}\left(X_{1}\right) \leq v_{1}, \ldots, F_{d}\left(X_{d}\right) \leq v_{d}\right)\right)$ depicts the dependence of the random vector $\left(X_{1}, \ldots, X_{d}\right)$. Tail dependence is a widely studied topic. Below we introduce the definitions of symmetric and tail dependence.

Definition 2 (Symmetry) For a given copula $C\left(v_{1}, \ldots, v_{d}\right)$, if

$$
C\left(v_{1}, \ldots, v_{i-1}, v_{i}, v_{i+1}, \ldots, v_{k-1}, v_{k}, v_{k+1}, \ldots, v_{d}\right)=C\left(v_{1}, \ldots, v_{i-1}, v_{k}, v_{i+1}, \ldots, v_{k-1}, v_{i}, v_{k+1}, \ldots, v_{d}\right),
$$

we say that $v_{i}$ and $v_{k}$ are exchangeable. For any pair $v_{i}, v_{k} \in \mathbf{I}$, if $v_{i}$ and $v_{k}$ are exchangeable, we say the copula $C\left(v_{1}, \ldots, v_{d}\right)$ is a symmetric copula.

Based on Definition 2, both the Clayton and the Gumbel copulas are symmetric.

Tail dependence coefficients provide useful information of the properties of the conditional probabilities of extreme values. Loosely speaking, the tail dependence describes the limiting property that one margin exceeds a certain threshold given that the other margin has already exceeded that threshold. For example, the lower tail dependence coefficient is the conditional probability that random vector $\left(F_{1}\left(X_{1}\right), \ldots, F_{d}\left(X_{d}\right)\right)$ with standard uniform margins belongs to the lower tail orthant given that a univariate margin takes extreme values.

Let $\triangleleft_{k}$ denote $\left\{X_{k} \leq F_{k}^{-}(v)\right\}$ and $\triangleright_{k}$ denote $\left\{X_{k}>F_{k}^{-}(1-v)\right\}, k=1, \ldots, d$. Then, $P\left(\triangleleft_{1}, \ldots, \triangleleft_{d}\right)=$ $C\left(v_{1}, \ldots, v_{d}\right)$. We can define the following tail dependence coefficients.

Definition 3 (Tail dependence) The lower-lower, lower-upper, upper-lower, and upper-upper tail dependence coefficients are defined below, respectively.

$$
\begin{aligned}
& \lambda_{i \mid k}^{l, l}(C)=\lim _{v \rightarrow 0+} P\left(\triangleleft_{1}, \ldots, \triangleleft_{k-1}, \triangleleft_{k+1}, \ldots, \triangleleft_{i-1}, \triangleleft_{i}, \triangleleft_{i+1}, \ldots, \triangleleft_{d} \mid \triangleleft_{k}\right), \\
& \lambda_{i \mid k}^{l, u}(C)=\lim _{v \rightarrow 0+} P\left(\triangleleft_{1}, \ldots, \triangleleft_{k-1}, \triangleleft_{k+1}, \ldots, \triangleleft_{i-1}, \triangleright_{i}, \triangleleft_{i+1}, \ldots, \triangleleft_{d} \mid \triangleleft_{k}\right), \\
& \lambda_{i \mid k}^{u, l}(C)=\lim _{v \rightarrow 0+} P\left(\triangleleft_{1}, \ldots, \triangleleft_{k-1}, \triangleleft_{k+1}, \ldots, \triangleleft_{i-1}, \triangleleft_{i}, \triangleleft_{i+1}, \ldots, \triangleleft_{d} \mid \triangleright_{k}\right), \\
& \lambda_{i \mid k}^{u, u}(C)=\lim _{v \rightarrow 0+} P\left(\triangleleft_{1}, \ldots, \triangleleft_{k-1}, \triangleleft_{k+1}, \ldots, \triangleleft_{i-1}, \triangleright_{i}, \triangleleft_{i+1}, \ldots, \triangleleft_{d} \mid \triangleright_{k}\right) .
\end{aligned}
$$


The above four coefficients define the amount of dependence in the tails of two variables in a multivariate distribution. For example, $\lambda_{i \mid k}^{l, u}(C)$ depicts the probability of the extreme values in the ( $k$-lower, $i$-upper) direction, or more precisely, the $i$-th variable is large given that the $k$-th variable is small.

Based on Definition 3, one can obtain the following Lemma.

Lemma 1 For a given copula $C\left(v_{1}, \ldots, v_{d}\right)$, if $v_{i}$ and $v_{k}$ are exchangeable, then $\lambda_{i \mid k}^{u, l}(C)=\lambda_{i \mid k}^{l, u}(C)$.

The proofs of the lemmas and the theorems in this paper can be found in the Appendix.

For example, for both the bivariate Clayton and the bivariate Gumbel copulas, $\lambda_{i \mid k}^{l, u}(C)=\lambda_{i \mid k}^{u, l}(C)$, but $\lambda_{i \mid k}^{l, l}(C) \neq \lambda_{i \mid k}^{u, u}(C)$.

Lemma 1 is important as it shows the following fact. If $v_{i}$ and $v_{k}$ are exchangeable in $C\left(v_{1}, \ldots, v_{d}\right)$, then the tail dependence coefficient in the ( $k$-lower, $i$-upper) direction and that in the ( $k$-upper, $i$-lower) direction are equal. This poses a problem that such a copula cannot fit data exhibiting unequal tail dependence in the two directions (see the end of Section 1.2.2 for a data example). To overcome this drawback, we introduce a method of constructing asymmetric copulas in the following section.

\subsection{Construction of asymmetric multivariate copulas}

Denote

$$
\breve{C}_{k}\left(v_{1}, \ldots, v_{d}\right)=C\left(v_{1}, \ldots, v_{k-1}, 1, v_{k+1}, \ldots, v_{d}\right)-C\left(v_{1}, \ldots, v_{k-1}, 1-v_{k}, v_{k+1}, \ldots v_{d}\right)
$$

Theorem 1 If $C\left(v_{1}, \ldots, v_{d}\right)$ is a copula, then $\breve{C}_{k}\left(v_{1}, \ldots, v_{d}\right)$ is a copula.

We may refer to $C\left(v_{1}, \ldots v_{d}\right)$ in $(7)$ as a base copula. From Theorem 1 , we can have the following Lemma.

Lemma 2 Theorem 1 suggests that the identity

$$
C\left(v_{1}, \ldots, v_{i-1}, v_{i}, v_{i+1}, \ldots, v_{k-1}, v_{k}, v_{k+1}, \ldots, v_{d}\right)=C\left(v_{1}, \ldots, v_{i-1}, v_{k}, v_{i+1}, \ldots, v_{k-1}, v_{i}, v_{k+1}, \ldots, v_{d}\right)
$$

does not entail

$$
\breve{C}_{k}\left(v_{1}, \ldots, v_{i-1}, v_{i}, v_{i+1}, \ldots, v_{k-1}, v_{k}, v_{k+1}, \ldots, v_{d}\right)=\breve{C}_{k}\left(v_{1}, \ldots, v_{i-1}, v_{k}, v_{i+1}, \ldots, v_{k-1}, v_{i}, v_{k+1}, \ldots, v_{d}\right)
$$

for all $v_{i} \in[0,1]$ 
In other words, although $v_{i}$ and $v_{k}$ are exchangeable in copula $C\left(v_{1}, \ldots, v_{d}\right)$, they may not be exchangeable in copula $\breve{C}_{k}\left(v_{1}, \ldots, v_{d}\right)$. As such, from Lemma 1 and Lemma $2, \breve{C}_{k}\left(v_{1}, \ldots, v_{d}\right)$ can be used to fit the data exhibiting unequal tail dependence in the direction along the ( $k$-lower, $i$-upper) and ( $k$-upper, $i$-lower), where $i=1, \ldots, k-1, k+1, \ldots, d$.

$$
\breve{C}_{k}\left(v_{1}, \ldots, v_{d}\right)=\left(\sum_{\substack{i \neq k \\ i=1}}^{d} v_{i}^{-\theta}\right)^{-1 / \theta}-\left[\left(1-v_{k}\right)^{-\theta}+\sum_{\substack{i \neq k \\ i=1}}^{d} v_{i}^{-\theta}-1\right]^{-1 / \theta} .
$$

If the $d$-dimensional Gumbel copula is used as a base copula, then

$$
\left.\breve{C}_{k}\left(v_{1}, \ldots, v_{d}\right)=\exp \left\{-\left[\sum_{\substack{i \neq k \\ i=1}}^{d}\left(-\ln v_{i}\right)^{\theta}\right]^{1 / \theta}\right\}-\exp \left\{-\left[\left(-\ln v_{k}\right)^{\theta}\right]^{1 / \theta}+\sum_{\substack{i \neq k \\ i=1}}^{d}\left(-\ln v_{i}\right)^{\theta}\right]^{1 / \theta}\right\} .
$$

It is apparent that the above two copulas (8) and (9) are asymmetric in the sense that $v_{i}$ is not exchangeable with other variables.

From Theorem 1, we have the following Lemma 3.

Lemma 3 From Theorem 1, we have

(a). Denote the density of $C\left(v_{1}, \ldots v_{d}\right)$ by $c\left(v_{1}, \ldots, v_{d}\right)$, then the density of copula $\breve{C}_{k}\left(v_{1}, \ldots, v_{d}\right)$ is $c\left(v_{1}, \ldots, v_{k-1}, 1-v_{k}, v_{k+1}, \ldots v_{d}\right)$.

(b). If $X_{k}$ is independent of the other variables $\left(X_{1}, \ldots, X_{k-1}, X_{k+1}, \ldots X_{d}\right)$, that is

$$
\begin{aligned}
& P\left(X_{1} \leq x_{1}, \ldots, X_{k-1} \leq x_{k-1}, X_{k+1} \leq x_{k+1}, \ldots, X_{d} \leq x_{d} \mid X_{k} \leq x_{k}\right) \\
= & P\left(X_{1} \leq x_{1}, \ldots, X_{k-1} \leq x_{k-1}, X_{k+1} \leq x_{k+1}, \ldots,, X_{d} \leq x_{d}\right),
\end{aligned}
$$

then $v_{k}$ in $\breve{C}_{k}\left(v_{1}, \ldots, v_{d}\right)$ is exchangeable with any other $v_{i}$ (with $i \in\{1, . ., k-1, k+1, \ldots, d\}$ ).

Point (b) of Lemma 3 suggests that, if the independence copula, $C\left(v_{1}, \ldots, v_{d}\right)=\prod_{k=1}^{d} v_{k}$, is the base copulas, for all $k=1, \ldots, d$, then $\breve{C}_{k}\left(v_{1}, \ldots, v_{d}\right)$ is not an asymmetric copula.

As can be seen in the following section, one of the merits of using $\breve{C}_{k}\left(v_{1}, \ldots, v_{d}\right)$ is its capability of capturing the tail dependence. As such, we can conclude that the method of construction of asymmetric copulas introduced in Theorem 1 differs from the Liebscher's method in [12] from the following aspects. The single-dimensional asymmetry method constructs copulas that present the asymmetric property in one variable (variable $k$ in Theorem 1, for example), whereas the Liebscher's method constructs copulas that may have the asymmetric property in all of the variables. Of course, when $d=2$, such difference does not exist.

To overcome the drawback that $\breve{C}_{k}\left(v_{1}, \ldots, v_{d}\right)$ only presents the asymmetry property in a single dimension along the variable $v_{k}$, one may use the following (11) to construct copulas with the asymmetry property in multiple directions. 
Lemma 4 Let

$$
\bar{C}\left(v_{1}, v_{2}, \ldots, v_{d}\right)=\sum_{k=0}^{d} p_{k} \breve{C}_{k}\left(v_{1}, v_{2}, \ldots, v_{d}\right),
$$

where $\sum_{k=0}^{d} p_{k}=1,0 \leq p_{k} \leq 1$ and $\breve{C}_{0}\left(v_{1}, v_{2}, \ldots, v_{d}\right)=C\left(v_{1}, v_{2}, \ldots, v_{d}\right)$, then $\bar{C}\left(v_{1}, v_{2}, \ldots, v_{d}\right)$ is a copula.

In (11), the parameters of different copula $\breve{C}_{k}\left(v_{1}, v_{2}, \ldots, v_{d}\right)$ with $k=1, . ., d$ can take different values. For example, if the Clayton copula shown in (1) is used, the parameter $\theta$ of $\breve{C}_{k}\left(v_{1}, v_{2}, \ldots, v_{d}\right)$ can take different values over different $k$ 's.

With (11), if one wants to build a copula in which the $i_{1}$ th, .., $i_{k}$ th variables are not exchangeable with other variables, he/she can let $p_{i_{1}} p_{i_{2}} \ldots p_{i_{k}}>0$. Hence, the copula can have unequal tail dependence coefficients along the $i_{1}$ th $, \ldots, i_{k}$ th directions. Examples of using such a method to construct the asymmetric bivariate copulas are given in Lemma 8.

$\breve{C}_{k_{1}, \ldots, k_{i}}\left(v_{1}, \ldots, v_{d}\right)=\breve{C}_{k_{1}, \ldots, k_{i-1}}\left(v_{1}, \ldots, v_{k_{i}-1}, 1, v_{k_{i}+1}, \ldots, v_{d}\right)-\breve{C}_{k_{1}, \ldots, k_{i}-1}\left(v_{1}, \ldots, v_{k_{i}-1}, 1-v_{k_{i}}, v_{k_{i}+1}, \ldots, v_{d}\right)$

The iteration formula (12) provides an alternative method to construct asymmetric copulas. For example, for a given copula $C\left(v_{1}, \ldots v_{4}\right)$, it possesses: $v_{1}$ and $v_{2}$ are exchangeable, and $v_{3}$ and $v_{4}$ are exchangeable. From Lemma 1, the copula has: $\lambda_{2 \mid 1}^{l, u}(C)=\lambda_{2 \mid 1}^{u, l}(C)$ and $\lambda_{4 \mid 3}^{l, u}(C)=\lambda_{4 \mid 3}^{u, l}(C)$. Assuming one wants to construct a new copula $C_{\text {new }}\left(v_{1}, \ldots v_{4}\right)$ with $\lambda_{2 \mid 1}^{l}\left(C_{\text {new }}\right) \neq \lambda_{2 \mid 1}^{u, l}\left(C_{\text {new }}\right)$ and $\lambda_{4 \mid 3}^{l, u}\left(C_{\text {new }}\right) \neq$ $\lambda_{4 \mid 3}^{u, l}\left(C_{n e w}\right)$, he can use (12) to construct the copula $C_{n e w}\left(v_{1}, v_{2}, v_{3}, v_{4}\right)=\breve{C}_{1,3}\left(v_{1}, v_{2}, v_{3}, v_{4}\right)$ that possesses $\lambda_{2 \mid 1}^{l, u}\left(C_{\text {new }}\right) \neq \lambda_{2 \mid 1}^{u, l}\left(C_{\text {new }}\right)$ and $\lambda_{4 \mid 3}^{l, u}\left(C_{\text {new }}\right) \neq \lambda_{4 \mid 3}^{u, l}\left(C_{\text {new }}\right)$.

The tail dependence coefficients defined in Definition 3 can be re-written in terms of copulas, as shown in Lemma 5.

Lemma 5 The tail dependence coefficients defined in Definition 3 can also be expressed as

$$
\begin{aligned}
& \lambda_{i \mid k}^{l, l}(C)=\lim _{v \rightarrow 0+} \frac{C(v, \ldots, v)}{v}, \\
& \lambda_{i \mid k}^{l, u}(C)=\lim _{v \rightarrow 0+} \frac{\breve{C}_{i}(v, \ldots, v)}{v}, \\
& \lambda_{i \mid k}^{u, l}(C)=\lim _{v \rightarrow 0+} \frac{\breve{C}_{k}(v, \ldots, v)}{v}, \\
& \lambda_{i \mid k}^{u, u}(C)=\lim _{v \rightarrow 0+} \frac{\breve{C}_{k, i}(v, \ldots, v)}{v},
\end{aligned}
$$

respectively, where $\breve{C}_{k, i}\left(v_{1}, \ldots, v_{d}\right)=\breve{C}_{k}\left(v_{1}, \ldots, v_{i-1}, 1, v_{i+1}, \ldots, v_{d}\right)-\breve{C}_{k}\left(v_{1}, \ldots, v_{i-1}, 1-v_{i}, v_{i+1}, \ldots, v_{d}\right)$.

From Lemma 5, we have the following lemma. 
Lemma 6 The tail dependence coefficients have the following relationships.

$$
\begin{aligned}
\lambda_{i \mid k}^{l, l}\left(\breve{C}_{i}\right) & =\lambda_{i \mid k}^{l, u}(C), \\
\lambda_{i \mid k}^{l, l}\left(\breve{C}_{k}\right) & =\lambda_{i \mid k}^{u, l}(C), \\
\lambda_{i \mid k}^{l, l}\left(\breve{C}_{k, i}\right) & =\lambda_{i \mid k}^{u, u}(C) .
\end{aligned}
$$

The reason that the above lemma holds is that the copulas $\breve{C}_{i}, \breve{C}_{k}$, and $\breve{C}_{k, i}$ are rotated copulas and differ from each other because they are constructed considering a different degree of rotation.

\subsection{Construction of bivariate asymmetric copulas}

Following Definition 1, a bivariate copula $C\left(v_{1}, v_{2}\right)$ can also be defined (see [3] for more detailed discussion).

One can have the tail dependence coefficients for the bivariate copula case as following.

Lemma 7 From Lemma 5, the tail dependence coefficients of copula $C\left(v_{1}, v_{2}\right)$ are given by

$$
\begin{aligned}
& \lambda_{2 \mid 1}^{l, l}(C)=\lim _{v \rightarrow 0+} P\left(X_{2} \leq F_{2}^{-}(v) \mid X_{1} \leq F_{1}^{-}(v)\right)=\lim _{v \rightarrow 0+} \frac{C(v, v)}{v}, \\
& \lambda_{2 \mid 1}^{l, u}(C)=\lim _{v \rightarrow 0+} P\left(X_{2}>F_{2}^{-}(1-v) \mid X_{1} \leq F_{1}^{-}(v)\right)=1-\lim _{v \rightarrow 0+} \frac{C(v, 1-v)}{v}, \\
& \lambda_{2 \mid 1}^{u, l}(C)=\lim _{v \rightarrow 0+} P\left(X_{2} \leq F_{2}^{-}(v) \mid X_{1}>F_{1}^{-}(1-v)\right)=1-\lim _{v \rightarrow 0+} \frac{C(1-v, v)}{v}, \\
& \lambda_{2 \mid 1}^{u, u}(C)=\lim _{v \rightarrow 0+} P\left(X_{2}>F_{2}^{-}(1-v) \mid X_{1}>F_{1}^{-}(1-v)\right)=2-\lim _{v \rightarrow 0+} \frac{1-C(1-v, 1-v)}{v} .
\end{aligned}
$$

Lemma 7 provides tail dependence coefficients in four different directions.

Some widely used copulas have either $\lambda_{2 \mid 1}^{u, u}(C)>0$ or $\lambda_{2 \mid 1}^{l, l}(C)>0$. For example, the Clayton copula has $\lambda_{2 \mid 1}^{l, l}(C)=2^{1 / \theta}$ and $\lambda_{2 \mid 1}^{u, u}(C)=0$ while the Gumbel copula has $\lambda_{2 \mid 1}^{u, u}(C)=2-2^{1 / \theta}$ and $\lambda_{2 \mid 1}^{l, l}(C)=0$. For both the Clayton copula and the Gumbel copula, $\lambda_{2 \mid 1}^{l, u}(C)=\lambda_{2 \mid 1}^{u, l}(C)=0$.

Following the notation in Section 2.3, for a given bivariate copula $C\left(v_{1}, v_{2}\right)$, we have

$$
\begin{aligned}
& \breve{C}_{1}\left(v_{1}, v_{2}\right)=v_{2}-C\left(1-v_{1}, v_{2}\right) \\
& \breve{C}_{2}\left(v_{1}, v_{2}\right)=v_{1}-C\left(v_{1}, 1-v_{2}\right)
\end{aligned}
$$


and

$$
\bar{C}\left(v_{1}, v_{2}\right)=p_{0} C\left(v_{1}, v_{2}\right)+p_{1} \breve{C}_{1}\left(v_{1}, v_{2}\right)+p_{2} \breve{C}_{2}\left(v_{1}, v_{2}\right)
$$

where $p_{k} \geq 0$ and $\sum_{k=0}^{2} p_{k}=1$.

The above copulas $\breve{C}_{k}\left(v_{1}, v_{2}\right)(k=1,2)$ and $\bar{C}\left(v_{1}, v_{2}\right)$ are also discussed in the monograph by Nelsen [3] for a different purpose. It is easy to prove the following lemma.

Lemma 8 For the copula $\bar{C}\left(v_{1}, v_{2}\right)$ defined in (26),

(a) we have

$$
\begin{aligned}
& \lambda_{2 \mid 1}^{l, l}(\bar{C})=p_{0} \lambda_{2 \mid 1}^{l, l}(C)+p_{1} \lambda_{2 \mid 1}^{u, l}(C)+p_{2} \lambda_{2 \mid 1}^{l, u}(C), \\
& \lambda_{2 \mid 1}^{l, u}(\bar{C})=p_{0} \lambda_{2 \mid 1}^{l, u}(C)+p_{1} \lambda_{2 \mid 1}^{u, u}(C)+p_{2} \lambda_{2 \mid 1}^{l, l}(C), \\
& \lambda_{2 \mid 1}^{u, l}(\bar{C})=p_{0} \lambda_{2 \mid 1}^{u, l}(C)+p_{1} \lambda_{2 \mid 1}^{l, l}(C)+p_{2} \lambda_{2 \mid 1}^{u, u}(C), \\
& \lambda_{2 \mid 1}^{u, u}(\bar{C})=p_{0} \lambda_{2 \mid 1}^{u, u}(C)+p_{1} \lambda_{2 \mid 1}^{l, u}(C)+p_{2} \lambda_{2 \mid 1}^{u, l}(C),
\end{aligned}
$$

(b) if $p_{1} p_{2}>0$, then $\lambda_{2 \mid 1}^{l, u}(\bar{C}) \lambda_{2 \mid 1}^{u, l}(\bar{C})>0$.

Remark 1 From point (a) of the above lemma, one can construct copulas with $\lambda_{2 \mid 1}^{l, u}(\bar{C}) \neq \lambda_{2 \mid 1}^{u, l}(\bar{C})$ and/or $\lambda_{2 \mid 1}^{l, l}(\bar{C}) \neq \lambda_{2 \mid 1}^{u, u}(\bar{C})$. From point (b), if $p_{1} p_{2}>0$, then both $\lambda_{2 \mid 1}^{l, u}(\bar{C})$ and $\lambda_{2 \mid 1}^{u, l}(\bar{C})$ are nonzeros. This result is useful as many popular copulas have either $\lambda_{2 \mid 1}^{l, l}(C) \neq 0$ or $\lambda_{2 \mid 1}^{u, u}(C) \neq 0$ but they have $\lambda_{2 \mid 1}^{l, u}=\lambda_{2 \mid 1}^{u, l}=0$.

For example, the Clayton copula has $\lambda_{2 \mid 1}^{l, l}(\bar{C})=2^{1 / \theta} p_{0}, \lambda_{2 \mid 1}^{l, u}(\bar{C})=2^{1 / \theta} p_{2}, \lambda_{2 \mid 1}^{u, l}(\bar{C})=2^{1 / \theta} p_{1}$, and $\lambda_{2 \mid 1}^{u, u}(\bar{C})=0$, while the Gumbel copula has $\lambda_{2 \mid 1}^{l, l}(\bar{C})=0, \lambda_{2 \mid 1}^{l, u}(\bar{C})=\left(2-2^{1 / \theta}\right) p_{1}, \lambda_{2 \mid 1}^{u, l}(\bar{C})=$ $\left(2-2^{1 / \theta}\right) p_{2}$, and $\lambda_{2 \mid 1}^{u, u}(\bar{C})=\left(2-2^{1 / \theta}\right) p_{0}$. As such, one can use $\bar{C}\left(v_{1}, v_{2}\right)$ to build new copulas with non-zero $\lambda_{2 \mid 1}^{l, u}(\bar{C})$ and/or $\lambda_{2 \mid 1}^{u, l}(\bar{C})$. See Section 3 that gives an example.

Let $C\left(v_{1}, v_{2}\right)$ be a bivariate Clayton copula, and $v_{1}$ and $v_{2}$ follow normal distributions $N\left(\mu_{1}, \sigma_{1}\right)$ and $N\left(\mu_{2}, \sigma_{2}\right)$, where $\mu_{1}=1, \sigma_{1}=1$, and $\mu_{2}=5, \sigma_{2}=3$, respectively. Figure 2.(a) shows the contour of the density of $C\left(v_{1}, v_{2}\right)$ with $\theta=0.8$. Figure 2.(b) shows the contour of the density of the copula $\bar{C}\left(v_{1}, v_{2}\right)=p_{0} C\left(v_{1}, v_{2}\right)+p_{1} \breve{C}_{1}\left(v_{1}, v_{2}\right)$ with $\theta=0.8, p_{0}=0.7$, and $p_{1}=0.3$. From Figure 2.(b), it can be seen there are tail dependence in the lower-lower area as well as in the upper-lower area.

As above-mentioned, the bivariate method used in modelling reliability data needs to select a bivariate distribution in which the two variables are positively correlated. Copulas provide a natural way to study and measure dependence between random variables. Linear correlation (or Pearsons correlation) is most frequently used in practice as a measure of dependence. The Kendall's tau and the Spearmans rho provide the perhaps best alternatives to the linear correla- 

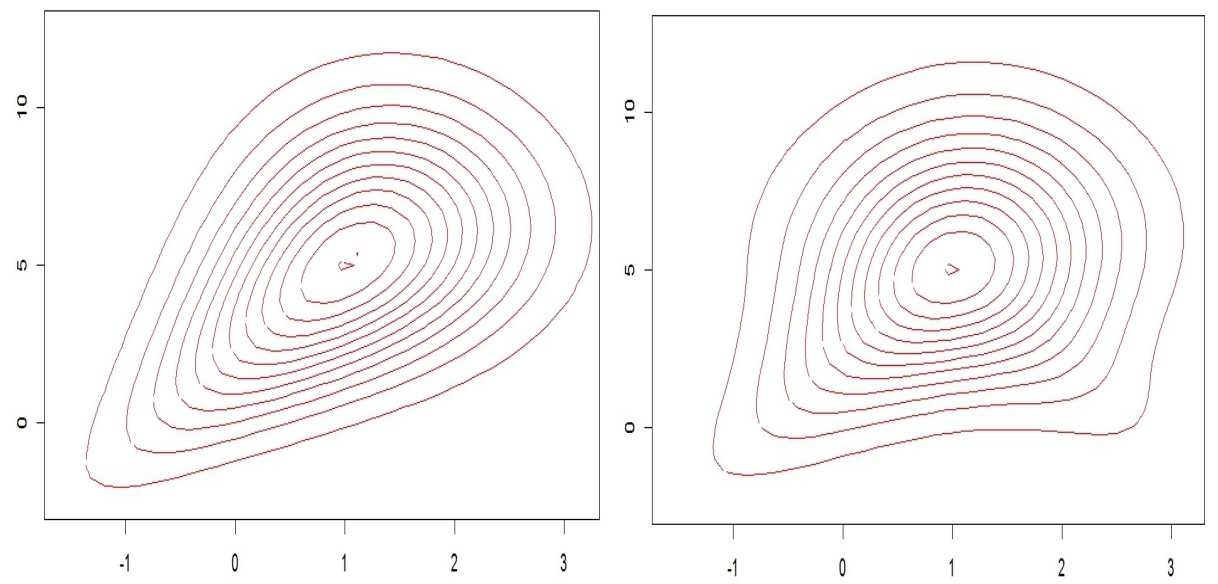

(a) The density of Clayton copula(b) The density of copula with $\theta=0.8$

$$
\begin{aligned}
& p_{0} C\left(v_{1}, v_{2}\right)+p_{1} \breve{C}_{1}\left(v_{1}, v_{2}\right) \quad \text { with } \\
& \theta=0.8, p_{0}=0.7 \text { and } p_{1}=0.3
\end{aligned}
$$

Fig. 2. Contours of the densities of the Clayton copulas

tion coefficient as a measure of dependence for nonelliptical distributions [3], for which the linear correlation coefficient is inappropriate and often misleading [24]. For example, the Kendall's tau of the Clayton copula in (1) and that of the Gumbel copula defined in (2) are $\frac{\theta}{2+\theta}$ and $1-\frac{1}{\theta}$, respectively. Unfortunately, the Spearman's rho's of the two copulas are too complicated to be given.

The Kendall's tau and the Spearman's rho of copula $\bar{C}\left(v_{1}, v_{2}\right)$ are discussed in the following lemma.

Lemma 9 For the copula $\bar{C}\left(v_{1}, v_{2}\right)$ defined in (26), if the Spearman's rho of $C\left(v_{1}, v_{2}\right)$ is $\rho_{0}$, then

(a) the Kendall's tau of $\bar{C}\left(v_{1}, v_{2}\right)$ satisfies $\frac{1}{3}\left(2\left(p_{0}-p_{1}-p_{2}\right) \rho_{0}-1\right) \leq \tau \leq \frac{1}{3}\left(2\left(p_{0}-p_{1}-p_{2}\right) \rho_{0}+1\right)$, and

(b) the Spearman's rho of $\bar{C}\left(v_{1}, v_{2}\right)$ is $\left(p_{0}-p_{1}-p_{2}\right) \rho_{0}$.

Lemma 9 suggests that both the Kendall's tau and the Spearman's rho are positive if $p_{0}-p_{1}-p_{2}>$ 0 in $\bar{C}\left(v_{1}, v_{2}\right)$.

\section{Application in two-dimensional reliability modelling}

As discussed above, modelling two-dimensional reliability data is important for both manufacturers and asset managers. In this section, we show how the proposed method of constructing asymmetric copulas can be applied in modelling reliability data.

Remark 2 With the above analysis, one can conclude that the age $X_{1}$ and the usage $X_{2}$ of reliability systems should satisfy the following two conditions. 
- Condition 1 (Positive dependence). $X_{1}$ and $X_{2}$ are positively correlated in the sense that their Kendall's tau and Spearmans rho are larger than zero; and

- Condition 2 (Tail dependence). If the copula associated with the joint probability distribution of $X_{1}$ and $X_{2}$ is $C\left(v_{1}, v_{2}\right)$, then $\lambda_{2 \mid 1}^{u, l}(C) \geq \lambda_{2 \mid 1}^{l, u}(C)$.

Condition 1 is needed because the usage of a system has usually a positive relationship with the age of the system. Condition 2 reflects the relationship between the extreme values of the age and the usage, as explained in bulleted points (a) and (b) in Section 1.2.2.

Let $V_{k}=F_{k}\left(X_{k}\right)$ where $k=1,2$. Condition 2 can also be interpreted as $\lim _{v \rightarrow 0+} \frac{P\left(V_{2} \leq v \mid V_{1}>1-v\right)}{P\left(V_{2}>1-v \mid V_{1} \leq v\right)}>$ 1 , which can also be expressed as

$$
\begin{aligned}
& \lim _{v \rightarrow 0+} \frac{P\left(V_{2} \leq v \mid V_{1}>1-v\right)}{P\left(V_{2}>1-v \mid V_{1} \leq v\right)} \\
& =\lim _{v \rightarrow 0+} \frac{P\left(V_{1}>1-v, V_{2} \leq v\right) P\left(V_{1} \leq v\right)}{P\left(V_{1} \leq v, V_{2}>1-v\right) P\left(V_{1}>1-v\right)} \\
& =\lim _{v \rightarrow 0+} \frac{\left[P\left(V_{2} \leq v\right)-P\left(V_{1} \leq 1-v, V_{2} \leq v\right] P\left(V_{1} \leq v\right)\right.}{\left[P\left(V_{1} \leq v\right)-P\left(V_{1} \leq v, V_{2} \leq 1-v\right)\right]\left[1-P\left(V_{1} \leq 1-v\right)\right]} \\
& =\lim _{v \rightarrow 0+} \frac{v-C(1-v, v)}{v-C(v, 1-v)}>1 .
\end{aligned}
$$

In the following, we will use the copula based method that satisfies the two conditions explained in Remark 2.

\subsection{Re-thinking of the existing two-dimensional reliability models}

Many bivariate distribution models have been proposed to model two-dimensional reliability data (see $[15,18,20,21]$, for example). It can be easily checked that some bivariate distributions cannot capture the existence of the upper-lower or the lower-upper tail dependence. Below we analyse the one proposed in reference [20] as an example.

Jung and Bai [20] proposed using the following bivariate survival distribution to fit two-dimensional reliability data.

$$
S\left(x_{1}, x_{2}\right)=\exp \left\{-\left[\left(\frac{x_{1}}{\beta_{1}}\right)^{\alpha_{1} \theta}+\left(\frac{x_{2}}{\beta_{2}}\right)^{\alpha_{2} \theta}\right]^{1 / \theta}\right\} .
$$

One can rewrite $(32)$ as

$$
S\left(x_{1}, x_{2}\right)=\exp \left\{-\left[\left(-\ln \left(1-v_{1}\right)\right)^{\theta}+\left(-\ln \left(1-v_{2}\right)\right)^{\theta}\right]^{1 / \theta}\right\},
$$

where $v_{1}=1-\exp \left\{-\left(\frac{x_{1}}{\beta_{1}}\right)^{\alpha_{1}}\right\}$ and $v_{2}=1-\exp \left\{-\left(\frac{x_{2}}{\beta_{2}}\right)^{\alpha_{2}}\right\}$. 
Since

$$
S\left(x_{1}, x_{2}\right)=P\left(X_{1}>x_{1}, X_{2}>x_{2}\right)=1-F_{1}\left(x_{1}\right)-F_{2}\left(x_{2}\right)+F\left(x_{1}, x_{2}\right),
$$

or

$$
F\left(x_{1}, x_{2}\right)=F_{1}\left(x_{1}\right)+F_{2}\left(x_{2}\right)-1+S\left(x_{1}, x_{2}\right),
$$

where $F_{1}\left(x_{1}\right)=P\left(X_{1} \leq x_{1}\right), F_{2}\left(x_{2}\right)=P\left(X_{2} \leq x_{2}\right)$, and $F\left(x_{1}, x_{2}\right)=P\left(X_{1} \leq x_{1}, X_{2} \leq x_{2}\right)$, then the associated copula of $F\left(x_{1}, x_{2}\right)$ is given by

$$
C\left(v_{1}, v_{2}\right)=v_{1}+v_{2}-1+\exp \left\{-\left[\left(-\ln \left(1-v_{1}\right)\right)^{\theta}+\left(-\ln \left(1-v_{2}\right)\right)^{\theta}\right]^{1 / \theta}\right\}
$$

It can be re-written as

$$
C\left(v_{1}, v_{2}\right)=v_{1}+v_{2}-1+C_{0}\left(1-v_{1}, 1-v_{2}\right)
$$

where $C_{0}\left(v_{1}, v_{2}\right)=\exp \left\{-\left[\left(-\ln \left(v_{1}\right)\right)^{\theta}+\left(-\ln \left(v_{2}\right)\right)^{\theta}\right]^{1 / \theta}\right\}$ and $C_{0}\left(v_{1}, v_{2}\right)$ is the bivariate Gumbel copula.

Jung and Bai [20] claimed that some bivariate distributions may not be appropriate for modelling the two-dimensional failure data; the two variables should be positively correlated. One may interpret the positive correlation as the Kendall's tau or the Spearman's rho being positive.

It can be found that $\lambda_{2 \mid 1}^{l, l}(C) \neq \lambda_{2 \mid 1}^{u, u}(C)$ and $\lambda_{2 \mid 1}^{l, u}(C)=\lambda_{2 \mid 1}^{u, l}(C)$, which implies that this copula can capture the unequal tail dependence along the lower-lower tail and the upper-upper tail, but it is unable capture the unequal tail dependence along the lower-upper tail or the upper-lower tail.

Compared with the marginal method proposed in Lawless et al [25] and the time-scale method [21], Jung and Bai's method [20] has its merits as it is simple to use and directly fits a bivariate distribution. Their method, however, has the following drawbacks:

- The selection of the age distribution and the usage distribution to form a bivariate distribution may be hard. For example, it is not easy to find a bivariate distribution with a discrete marginal and a continuous marginal.

- As shown from the above analysis, their method cannot capture the asymmetric along the upper-lower tail dependency or the lower-upper tail dependency.

With copulas, the above drawbacks can be overcome. In the next section, we fit two-dimensional reliability data with such a copula.

\subsection{A case study}

In this section, we fit three copulas to a two-dimensional warranty claim data. 
In warranty management, product manufacturers can only collect warranty claim data on those products whose warranty is claimed. Product performance is not known on those sold products whose warranty is not claimed, or those data are censored.

\subsubsection{The data}

3,466 car warranty claims were collected from a car manufacturer. In those observations, the warranty of 2,289 cars were claimed within 36 months or 30,000 miles and the rest 1,177 cars were not claimed.

Figure 3.(a) and Figure 3.(b) show the histograms of the age and mileage of the claimed cars, respectively.

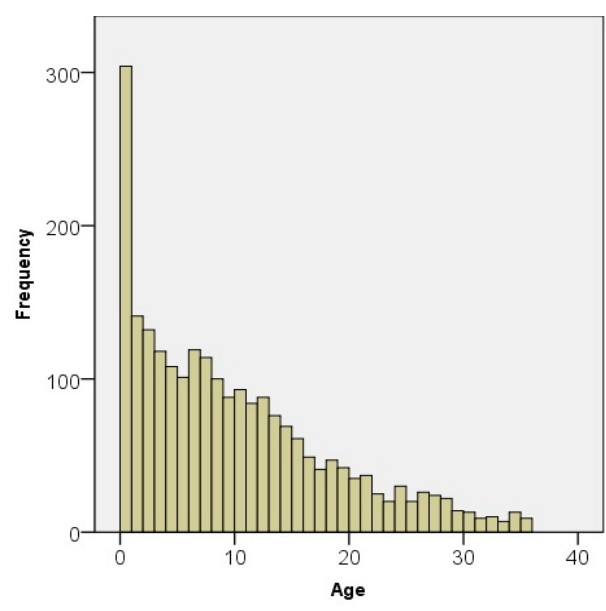

(a) Histogram of age

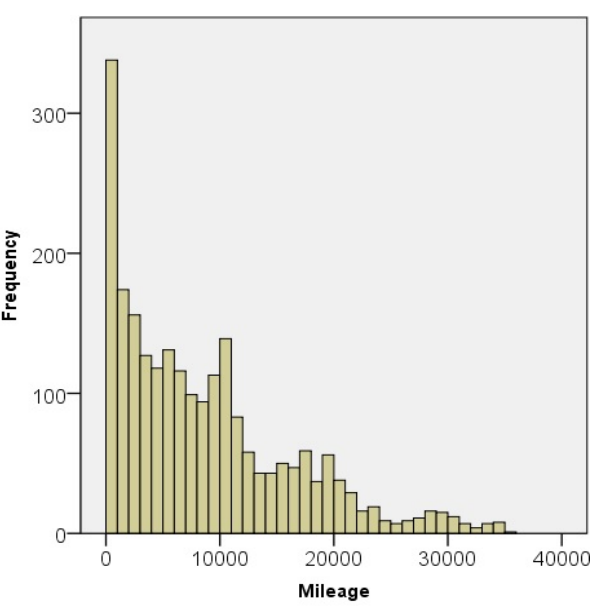

(b) Histogram of Usage (ie., Mileage)

Fig. 3. Histograms of the age and usage of the claimed cars.

\subsubsection{Modelling}

In order to compare the modelling method proposed by Jung and Bai [20], we use $C\left(v_{1}, v_{2}\right)(=$ $\left.v_{1}+v_{2}-1+C_{0}\left(1-v_{1}, 1-v_{2}\right)\right)$ in (37) as the base copula and use the following three models, where $C_{0}\left(v_{1}, v_{2}\right)=\exp \left\{-\left[\left(-\ln \left(v_{1}\right)\right)^{\theta}+\left(-\ln \left(v_{2}\right)\right)^{\theta}\right]^{1 / \theta}\right\}$.

Model 1. The copula is

$$
\begin{aligned}
C_{1}\left(v_{1}, v_{2}\right)= & p_{0} C\left(v_{1}, v_{2} ; \theta_{1}\right)+p_{1} \breve{C}_{1}\left(v_{1}, v_{2} ; \theta_{2}\right) \\
= & p_{0}\left(v_{1}+v_{2}-1+C_{0}\left(1-v_{1}, 1-v_{2} ; \theta_{1}\right)\right)+p_{1}\left(v_{1}-C_{0}\left(v_{1}, 1-v_{2} ; \theta_{2}\right)\right) \\
= & p_{0}\left\{v_{1}+v_{2}-1+\exp \left\{-\left[\left(-\ln \left(1-v_{1}\right)\right)^{\theta_{1}}+\left(-\ln \left(1-v_{2}\right)\right)^{\theta_{1}}\right]^{1 / \theta_{1}}\right\}\right\} \\
& +p_{1}\left\{v_{1}-\exp \left\{-\left[\left(-\ln \left(v_{1}\right)\right)^{\theta_{2}}+\left(-\ln \left(1-v_{2}\right)\right)^{\theta_{2}}\right]^{1 / \theta_{2}}\right\}\right\} .
\end{aligned}
$$

Model 2. The copula is 


$$
\begin{aligned}
C_{2}\left(v_{1}, v_{2}\right)= & p_{0} C\left(v_{1}, v_{2} ; \theta_{1}\right)+p_{1} C\left(v_{1}, v_{2} ; \theta_{2}\right) \\
= & p_{0}\left(v_{1}+v_{2}-1+C_{0}\left(1-v_{1}, 1-v_{2} ; \theta_{1}\right)\right)+p_{1}\left(v_{1}+v_{2}-1+C_{0}\left(1-v_{1}, 1-v_{2} ; \theta_{2}\right)\right) \\
= & v_{1}+v_{2}-1+p_{0}\left\{\exp \left\{-\left[\left(-\ln \left(1-v_{1}\right)\right)^{\theta_{1}}+\left(-\ln \left(1-v_{2}\right)\right)^{\theta_{1}}\right]^{1 / \theta_{1}}\right\}\right\} \\
& +p_{1}\left\{\exp \left\{-\left[\left(-\ln \left(1-v_{1}\right)\right)^{\theta_{2}}+\left(-\ln \left(1-v_{2}\right)\right)^{\theta_{2}}\right]^{1 / \theta_{2}}\right\}\right\}
\end{aligned}
$$

Model 3. The copula is

$$
\begin{aligned}
C_{3}\left(v_{1}, v_{2}\right) & =C\left(v_{1}, v_{2} ; \theta_{1}\right) \\
& =v_{1}+v_{2}-1+C_{0}\left(1-v_{1}, 1-v_{2} ; \theta_{1}\right) \\
& =v_{1}+v_{2}-1+\exp \left\{-\left[\left(-\ln \left(1-v_{1}\right)\right)^{\theta_{1}}+\left(-\ln \left(1-v_{2}\right)\right)^{\theta_{1}}\right]^{1 / \theta_{1}}\right\} .
\end{aligned}
$$

Model 1 is based on the method proposed in this paper. Model 2 is a mixture of two Gumbel copulas with different parameters $\theta_{1}$ and $\theta_{2}$. In both Model 1 and Model $2, p_{0}+p_{1}=1$. Model 3 is the model proposed in [20].

All of the above three models, Models 1, 2 and 3, use the copula $C(.,$.$) as the base copula. Hence,$ checking with Condition 2 in Remark 2, we have the following results.

- Model 1 has $\lambda_{2 \mid 1}^{l, l}\left(C_{1}\right)=p_{0}\left(2-2^{1 / \theta_{1}}\right), \lambda_{2 \mid 1}^{l, u}\left(C_{1}\right)=0, \lambda_{2 \mid 1}^{u, l}\left(C_{1}\right)=p_{1}\left(2-2^{1 / \theta_{2}}\right)$, and $\lambda_{2 \mid 1}^{u, u}\left(C_{1}\right)=0$. We have $\lambda_{2 \mid 1}^{u, l}(C)>\lambda_{2 \mid 1}^{l, u}(C)$ as long as $p_{0} \neq 1$. Hence Model 1 meets Condition 2.

- Model 2 does not meet Condition 2 as it has $\lambda_{2 \mid 1}^{l, l}\left(C_{2}\right)=2-2^{1 / \theta_{1}} p_{0}-2^{1 / \theta_{2}} p_{1}$, and $\lambda_{2 \mid 1}^{l, u}\left(C_{2}\right)=$ $\lambda_{2 \mid 1}^{u, l}\left(C_{2}\right)=\lambda_{2 \mid 1}^{u, u}\left(C_{2}\right)=0$.

- Model 3 does not meet Condition 2 as it has $\lambda_{2 \mid 1}^{l, l}\left(C_{3}\right)=2-2^{1 / \theta_{1}}$, and $\lambda_{2 \mid 1}^{l, u}\left(C_{3}\right)=\lambda_{2 \mid 1}^{u, l}\left(C_{3}\right)=$ $\lambda_{2 \mid 1}^{u, u}\left(C_{3}\right)=0$.

We maximise the following log-likelihood to estimate the parameters of the models.

$$
\begin{aligned}
L_{k}(\theta) & =\sum_{i \in D} \log f_{k}\left(x_{1}, x_{2}\right)+\sum_{i \notin D} \log \left(1-F_{k}\left(A_{w}, U_{w}\right)\right) \\
& =\sum_{i \in D} \log \left[f_{1}\left(x_{1}\right) f_{2}\left(x_{2}\right) c_{k}\left(F_{1}\left(x_{1}\right), F_{2}\left(x_{2}\right)\right)\right]+\left(N-N_{r}\right) \log \left[1-C_{k}\left(F_{1}\left(A_{w}\right), F_{2}\left(U_{w}\right)\right)\right]
\end{aligned}
$$

where $k=1,2,3, D=\left(0, A_{w}\right] \times\left(0, U_{w}\right], f_{k}\left(x_{1}, x_{2}\right)=f_{1}\left(x_{1}\right) f_{2}\left(x_{2}\right) c_{k}\left(v_{1}, v_{2}\right), c_{k}\left(v_{1}, v_{2}\right)=\frac{\partial^{2} C_{k}\left(v_{1}, v_{2}\right)}{\partial v_{1} \partial v_{2}}$, and $N-N_{r}$ is the number of cars that were not claimed. $A_{w}$ is the age limit and $U_{w}$ is the usage limit, here $A_{w}=36$ and $U_{w}=30,000$.

In (41), the component $\sum_{i \in D} \log f_{k}\left(x_{1}, x_{2}\right)$ is the log-likelihood for all of the claimed cars, and the component $\sum_{i \notin D} \log \left(1-F_{k}\left(A_{w}, U_{w}\right)\right)$ is the log-likelihood for all of those cars that had not been claimed within the region $\left(0, A_{w}\right] \times\left(0, U_{w}\right]$.

In the literature, there are some models, including the generalized Pareto distribution, the lognormal distribution, and the Weibull distribution, that were proposed as the marginal distributions 
for two-dimensional reliability modelling. We have tried to fit those distributions on the data and found that using the Weibull distribution as the marginals yields the smallest AIC (Akaike information criterion). As such, the Weibull distribution is adopted as the marginals. Denote $v_{1}=F_{1}\left(x_{1}\right)=1-\exp \left\{-\left(\frac{x_{1}}{\beta_{1}}\right)^{\alpha_{1}}\right\}$ and $v_{2}=F_{2}\left(x_{2}\right)=1-\exp \left\{-\left(\frac{x_{2}}{\beta_{2}}\right)^{\alpha_{2}}\right\}$.

We estimate the parameters of the above three models based on the original dataset (with 3466 observations), and obtain the following parameters. From Table 1, it can be seen that Model 1 Table 1

Parameters and performance of the three models.

\begin{tabular}{cccccccc|c}
\hline \hline$\alpha_{1}$ & $\beta_{1}$ & $\alpha_{2}$ & $\beta_{2}$ & $\theta_{1}$ & $\theta_{2}$ & $p_{0}$ & AIC & Methods \\
\hline 0.78 & 27.00 & 0.77 & 24496.98 & 3.76 & 0.48 & 0.90 & 30945.39 & Model 1 \\
0.73 & 33.38 & 0.65 & 37332.16 & 4.62 & 1.86 & 0.75 & 31061.48 & Model 2 \\
0.81 & 25.01 & 0.71 & 24965.02 & 3.01 & & & 31035.36 & Model 3 \\
\hline
\end{tabular}

outperforms Model 2 and Model 3 as the AIC value of Model 1 (i.e., 30945.30) is smaller than those of models 2 and 3.

Furthermore, in order to compare model performance on datasets with different samples, we build models on the following three cases of datasets. We randomly sample 500, 1500, and 3000 observations from the original dataset, which creates three datasets, denoted as $D_{1}, D_{2}$ and $D_{3}$, respectively. Some basic statistics of the claimed products in the three datasets are shown in Table 2, in which $N$ is the sample size and $N_{r}$ is the number of claimed observations. Of course, the number of the un-claimed observations is $N-N_{r}$. To reduce the uncertainty caused by the random sampling, we further sample with replacement for 20 times from each of the three datasets. This creates 20 datasets with sample size $N=500,1500$ and 3000, respectively, and obtains 60 datasets in total. Denote $D_{k, 1}, \ldots, D_{k, 20}$ as the 20 datasets sampled from dataset $D_{k}$, where $k=1,2,3$. The models will be built on each of the 60 datasets.

We calculate the Kendall's tau and the Spearman's rho based on the observations in each dataset $D_{k, i}$ with $k=1,2,3$ and $i=1,2, \ldots, 20$. The values in the 3 rd and the 4 th columns in Table 3 show the mean and the standard deviation of the Kendall's tau's and the Spearman's rho's on datasets $D_{k, 1}, \ldots, D_{k, 20}(k=1,2,3)$, respectively. From the table, we can see that both Kendall's tau and Spearman's rho are positive.

We estimate the parameters of Model 1, Model 2, and Model 3 based on dataset $D_{1,1}, \ldots, D_{1,20}$, $D_{2,1}, \ldots, D_{2,20}, D_{3,1}, \ldots, D_{3,20}$, respectively. The results are shown in Table 4 . The values without brackets are the averages of the parameter estimates, and the values within brackets are the standard deviations of the parameter estimates. For example, the underlined value 0.79 located in Cell(2,2) (ie., the 2nd row and 2nd column) and the value 0.032 within the brackets under it are the average value and the standard deviation of the 20 estimates of $\alpha_{1}$ based on datasets $D_{1,1}, \ldots, D_{1,20}$, respectively. Similarly, another underlined value 26.68 located in Cell $(8,3)$ and the 
Table 2

Basic statistics of the age and the usage of the claimed cars.

\begin{tabular}{|c|c|c|c|c|c|c|c|}
\hline Dataset & $N$ & $N_{r}$ & Age/Usage & Mean & Std. Deviation & Skewness & Kurtosis \\
\hline \multirow{2}{*}{$D_{1}$} & \multirow{2}{*}{500} & \multirow{2}{*}{324} & Age & 10.28 & 8.25 & 0.70 & -0.31 \\
\hline & & & Usage & 8890.70 & 7953.59 & 0.99 & 0.33 \\
\hline \multirow{2}{*}{$D_{2}$} & \multirow{2}{*}{1500} & \multirow{2}{*}{981} & Age & 9.78 & 8.33 & 1.06 & 0.60 \\
\hline & & & Usage & 8520.89 & 7475.36 & 1.13 & 0.86 \\
\hline \multirow{2}{*}{$D_{3}$} & \multirow{2}{*}{3000} & \multirow{2}{*}{1939} & Age & 10.21 & 8.44 & 0.90 & 0.13 \\
\hline & & & Usage & 8842.91 & 7628.02 & 1.05 & 0.66 \\
\hline
\end{tabular}

Table 3

Kendall's tau and Spearman's Rho

\begin{tabular}{c|c|c|c}
\hline \hline Data & $N$ & Kendall's tau & Spearman's rho \\
\hline$D_{1,1}, \ldots, D_{1,20}$ & 500 & $\begin{array}{c}0.615 \\
(0.024)\end{array}$ & $\begin{array}{c}0.796 \\
(0.024)\end{array}$ \\
\hline$D_{2,1}, \ldots, D_{2,20}$ & 1500 & $\begin{array}{c}0.635 \\
(0.012)\end{array}$ & $\begin{array}{c}0.819 \\
(0.012)\end{array}$ \\
\hline$D_{3,1}, \ldots, D_{3,20}$ & \multirow{2}{*}{3000} & $\begin{array}{c}0.631 \\
(0.011)\end{array}$ & 0.817 \\
\hline \hline
\end{tabular}

value 1.61 within the brackets under it are the average value and the standard deviation of the 20 estimates of $\beta_{1}$ based on datasets $D_{2,1}, \ldots, D_{2,20}$, respectively. The number 4469.18 in bold font in Cell $(2,9)$ (ie., the 2 nd row and 9th column) is the average of the AIC values of Model 1 on the 20 datasets $D_{1,1}, \ldots, D_{1,20}$, and the value 124.89 under this average value is the standard deviation of the AIC values.

From Table 4, we have the following findings.

(a). As can be seen from the last two columns, Model 1, which is the model proposed in this paper, always outperforms the other two models as it has the smallest AIC. Model 2 is the second best model, and Model 3 performs the worst.

(b). If we define a relative improvement factor as

$$
\frac{\text { Model 2's } A I C-\text { Model 1's } A I C}{\text { Model } 3^{\prime} s A I C-M o d e l 1^{\prime} s A I C},
$$


then for the cases $N=500,1500$ and 3000, their relative improvement factors are 0.130 $\left(=\frac{4471.50-4469.18}{4486.98-4469.18}\right), 0.205\left(=\frac{13455.11-13442.77}{13483.00-13422.77}\right)$ and $0.615\left(=\frac{26953.39-26919.03}{26974.92-26919.03}\right)$, respectively. This shows: the performance of Model 1 becomes increasingly better when the sample size becomes larger.

Table 4

Parameters and performance of the three models.

\begin{tabular}{|c|c|c|c|c|c|c|c|c|c|}
\hline$N$ & $\alpha_{1}$ & $\beta_{1}$ & $\alpha_{2}$ & $\beta_{2}$ & $\theta_{1}$ & $\theta_{2}$ & $p_{0}$ & $\mathrm{AIC}$ & Methods \\
\hline \multirow{6}{*}{500} & $\underline{0.79}$ & 26.79 & 0.79 & 23656.14 & 3.77 & 0.46 & 0.89 & 4469.18 & Model 1 \\
\hline & $(0.032)$ & $(2.72)$ & $(0.041)$ & $(2988.96)$ & $(0.28)$ & $(0.027)$ & $(0.029)$ & $(124.89)$ & \\
\hline & 0.78 & 26.39 & 0.74 & 24071.96 .11 & 2.26 & 3.50 & 0.42 & 4471.50 & Model 2 \\
\hline & $(0.056)$ & $(2.68)$ & $(0.067)$ & $(2618.457)$ & $(1.53)$ & $(2.10)$ & $(0.27)$ & $(123.53)$ & \\
\hline & 0.81 & 25.13 & 0.72 & 24268.27 & 2.88 & & & 4486.98 & Model 3 \\
\hline & $(0.033)$ & $(2.45)$ & $(0.043)$ & $(2381.91)$ & $(0.16)$ & & & (126.39) & \\
\hline \multirow{6}{*}{1500} & 0.77 & 26.68 & 0.76 & 24017.97 & 3.90 & 0.54 & 0.92 & 13442.77 & Model 1 \\
\hline & $(0.018)$ & (1.61) & $(0.036)$ & $(1346.64)$ & $(0.30)$ & $(0.19)$ & $(0.033)$ & $(198.83)$ & \\
\hline & 0.75 & 25.15 & 0.71 & 23237.66 & 3.65 & 2.74 & 0.58 & 13455.11 & Model 2 \\
\hline & $(0.024)$ & $(1.62)$ & $(0.025)$ & $(1798.94)$ & $(1.35)$ & $(1.48)$ & $(0.23)$ & (191.18) & \\
\hline & 0.79 & 24.16 & 0.71 & 23544.67 & 3.13 & & & 13483.00 & Model 3 \\
\hline & $(0.022)$ & $(1.63)$ & $(0.026)$ & (1678.95) & $(0.083)$ & & & (190.87) & \\
\hline \multirow{6}{*}{3000} & 0.77 & 30.23 & 0.75 & 29308.12 & 3.78 & 0.77 & 0.87 & 26919.03 & Model 1 \\
\hline & $(0.021)$ & $(3.26)$ & $(0.052)$ & $(5412.91)$ & $(0.38)$ & $(0.66)$ & $(0.21)$ & $(339.52)$ & \\
\hline & 0.75 & 30.38 & 0.69 & 31128.44 & 4.62 & 2.09 & 0.67 & 26953.39 & Model 2 \\
\hline & $(0.024)$ & $(4.30)$ & $(0.030)$ & $(6455.89)$ & $(0.89)$ & $(0.59)$ & $(0.14)$ & $(327.83)$ & \\
\hline & 0.79 & 24.69 & 0.73 & 22882.41 & 3.01 & & & 26974.92 & Model 3 \\
\hline & $(0.017)$ & (1.67) & $(0.016)$ & $(2942.32)$ & $(0.10)$ & & & $(350.35)$ & \\
\hline
\end{tabular}

\section{Conclusion}

This paper introduced a new method to construct asymmetric copulas. This method has the merit that it can construct asymmetric copulas with tail dependence of given directions. Using the proposed copula, the paper proposed a novel method to model two dimensional reliability data. It then tested the proposed method based on a warranty dataset collected from a car manufacturer. It was shown that the proposed method outperformed two existing modelling methods. 
Our future work aims to construct asymmetric copulas that possess other types of dependence, for example, to reflect the probability of the extreme values of a subset of random variables. Another possible research focus is to investigate the use of nonparametric or semiparametric copulas in reliability data modelling.

\section{Acknowledgements}

The author is indebted to the two reviewers for their constructive comments.

\section{References}

[1] A. Sklar, Fonctions de répartition àn dimensions et leurs marges, Publications de l'Institut de Statistique de l'Université de Paris 8 (1959) 229-231.

[2] H. Joe, Multivariate Models and Dependence Concepts, Monographs on Statistics and Applied Probability, 73, Chapman \& Hall, London, 1996.

[3] R. Nelsen, An Introduction to Copulas, Springer, New York, 1999.

[4] N. Kolev, U. Dos Anjos, B. V. D. M. Mendes, Copulas: A review and recent developments, Stochastic Models 22 (4) (2006) 617-660.

[5] C. Genest, B. Remillard, D. Beaudoin, Goodness-of-fit tests for copulas: A review and a power study, Insurance: Mathematics and Economics 44 (2) (2009) 199-213.

[6] P. Embrechts, Copulas: A personal view, Journal of Risk and Insurance 76 (3) (2009) 639-650.

[7] M. Zhang, Modelling total tail dependence along diagonals, Insurance: Mathematics and Economics 42 (1) (2008) 73-80.

[8] L. Hua, H. Joe, Tail order and intermediate tail dependence of multivariate copulas, Journal of Multivariate Analysis 102 (10) (2011) 1454-1471.

[9] P. Grundke, S. Polle, Crisis and risk dependencies, European Journal of Operational Research 223 (2) (2012) 518-528.

[10] W. Ye, X. Liu, B. Miao, Measuring the subprime crisis contagion: Evidence of change point analysis of copula functions, European Journal of Operational Research 222 (1) (2012) 96-103.

[11] A. Alfonsi, D. Brigo, New families of copulas based on periodic functions, Communications in Statistics - Theory and Methods 34 (7) (2005) 1437-1447.

[12] E. Liebscher, Construction of asymmetric multivariate copulas, Journal of Multivariate Analysis 99 (2008) 2234-2250. 
[13] S. Wu, Warranty data analysis: A review, Quality and Reliability Engineering International 28 (8) (2012) 795-805.

[14] S. Wu, A review on coarse warranty data and analysis, Reliability Engineering and System Safety 114 (1) (2013) 1-11.

[15] S. Wu, A. Akbarov, Support vector regression for warranty claim forecasting, European Journal of Operational Research 213 (1) (2011) 196-204.

[16] S. Wu, A. Akbarov, Forecasting warranty claims for recently launched products, Reliability Engineering and System Safety 106 (2012) 160-164.

[17] S. Anastasiadis, B. Anderson, S. Chukova, Auto warranty and driving patterns, Reliability Engineering and System Safety 116 (2013) 126-134.

[18] J. F. Lawless, M. J. Crowder, K. Lee, Analysis of reliability and warranty claims in products with age and usage scales, Technometrics 51 (1) (2009) 14-24.

[19] M. M. Alam, K. Suzuki, Lifetime estimation using only failure information from warranty database, IEEE Transactions on Reliability 58 (4) (2009).

[20] M. Jung, D. S. Bai, Analysis of field data under two-dimensional warranty, Reliability Engineering and System Safety 92 (2) (2007) 135-143.

[21] I. B. Gertsbakh, K. B. Kordonsky, Parallel time scales and two-dimensional manufacturer and individual customer warranties, IIE Transactions (Institute of Industrial Engineers) 30 (12) (1998) $1181-1189$.

[22] B. Rai, N. Singh, Modeling and analysis of automobile warranty data in presence of bias due to customer-rush near warranty expiration limit, Reliability Engineering and System Safety 86 (1) (2004) 83-94.

[23] T. Vijverberg, J. C. Winterwerp, S. G. J. Aarninkhof, H. Drost, Fine sediment dynamics in a shallow lake and implication for design of hydraulic works, Ocean Dynamics 61 (2-3) (2011) 187-202.

[24] P. Embrechts, F. Lindskog, A. McNeil, Modelling dependence with copulas and applications to risk management, Handbook of heavy tailed distributions in finance 8 (2003) 329-384.

[25] J. F. Lawless, X. J. Hu, J. Cao, Methods for the estimation of failure distributions and rates from automobile warranty data, Lifetime Data Analysis 1 (3) (1995) 227-240.

[26] D. Drouet-Mari, S. Kotz, Correlation and Dependence, Imperial College Press, London, 2001.

[27] W. Kruskal, Ordinal measures of association, Journal of the American Statistical Association 53 (1958) 814-861. 


\section{Appendix}

Proofs of Lemmas 1, 2, 4, 6 7, and 8. Proofs of those lemmas are trivial and are therefore omitted.

Proof of Theorem 1. Denote $V_{k}=F_{k}\left(X_{k}\right)$, where $k=1, \ldots, d$. Then

$$
\begin{aligned}
& P\left\{V_{1} \leq v_{1}, \ldots, V_{k-1} \leq v_{k-1}, 1-V_{k} \leq v_{k}, V_{k+1} \leq v_{k+1}, \ldots, V_{d} \leq v_{d}\right\} \\
= & P\left\{V_{1} \leq v_{1}, \ldots, V_{k-1} \leq v_{k-1}, V_{k}>1-v_{k}, V_{k+1} \leq v_{k+1}, \ldots, V_{d} \leq v_{d}\right\} \\
= & P\left\{V_{1} \leq v_{1}, \ldots, V_{k-1} \leq v_{k-1}, V_{k+1} \leq v_{k+1}, \ldots, V_{d} \leq v_{d}\right\} \\
& -P\left\{V_{1} \leq v_{1}, \ldots, V_{k-1} \leq v_{k-1}, V_{k} \leq 1-v_{k}, V_{k+1} \leq v_{k+1}, \ldots, V_{d} \leq v_{d}\right\} \\
= & C\left(v_{1}, \ldots, v_{k-1}, 1, v_{k+1}, \ldots, v_{d}\right)-C\left(v_{1}, \ldots, v_{k-1}, 1-v_{k}, v_{k+1}, \ldots, v_{d}\right) . \\
= & \breve{C}_{k}\left(v_{1}, \ldots, v_{d}\right)
\end{aligned}
$$

This proves that $\breve{C}_{k}\left(v_{1}, \ldots, v_{d}\right)$ is a copula.

Proof of Lemma 3 Proof of (a) in Lemma 3 is trivial and is therefore omitted. Below we prove (b) and (c), respectively.

- Proof of (b) of Lemma 3. If $X_{k}$ is independent of the rest variables $\left(X_{1}, \ldots, X_{k-1}, X_{k+1}, \ldots X_{d}\right)$, then

$$
C\left(v_{1}, \ldots, v_{d}\right)=v_{k} G\left(v_{1}, \ldots, v_{k-1}, v_{k+1}, \ldots, v_{d}\right)
$$

where $G($.$) is a function. Substituting C\left(v_{1}, \ldots, v_{d}\right)$ into (7), one can obtain (b) in Lemma 3 . Proof of Lemma 5. Denote $\omega_{k}=v$ and $\varpi_{k}=1-v$ with $k=1, \ldots, d$. We establish (14). The proofs of (13), (15), and (16) are similar.

$$
\begin{aligned}
\lambda_{i \mid k}^{l, u} & =\lim _{v \rightarrow 0+} P\left(\triangleleft_{1}, \ldots, \triangleleft_{k-1}, \triangleleft_{k+1}, \ldots, \triangleleft_{i-1}, \triangleright_{i}, \triangleleft_{i+1} \ldots, \triangleleft_{d} \mid \triangleleft_{k}\right) \\
& =\lim _{v \rightarrow 0+} \frac{P\left(\triangleleft_{1}, \ldots, \triangleleft_{k-1}, \triangleleft_{k}, \triangleleft_{k+1}, \ldots, \triangleleft_{i-1}, \triangleright_{i}, \triangleleft_{i+1} \ldots, \triangleleft_{d}\right)}{P\left(\triangleleft_{k}\right)} \\
& =\lim _{v \rightarrow 0+} \frac{P\left(\triangleleft_{1}, \ldots, \triangleleft_{k-1}, \triangleleft_{k}, \triangleleft_{k+1}, \ldots, \triangleleft_{i-1}, \triangleleft_{i+1} \ldots, \triangleleft_{d}\right)-P\left(\triangleleft_{1}, \ldots, \triangleleft_{k-1}, \triangleleft_{k}, \triangleleft_{k+1}, \ldots, \triangleleft_{i-1}, \triangleleft_{i}^{\prime}, \triangleleft_{i+1} \ldots, \triangleleft_{d}\right)}{P\left(\triangleleft_{k}\right)} \\
& =\lim _{v \rightarrow 0+} \frac{C\left(\omega_{1}, \ldots, \omega_{i-1}, 1, \omega_{i+1} \ldots, \omega_{d}\right)-C\left(\omega_{1}, \ldots, \omega_{i-1}, \varpi_{i}, \omega_{i+1} \ldots, \omega_{d}\right)}{v} \\
& =\lim _{v \rightarrow 0+} \frac{\breve{C}_{i}\left(\omega_{1}, \ldots, \omega_{d}\right)}{v}
\end{aligned}
$$

where $\triangleleft_{i}^{\prime}=\left\{X_{i} \leq F_{i}^{-}(1-v)\right\}$.

Proof of Lemma 9. The proof of Lemma 9 can easily be done based on the following concepts.

Let $X_{1}$ and $X_{2}$ be continuous random variables whose copula is $C($.$) . Then the population$ version Spearman's rho (or $\rho$ ) between $X_{1}$ and $X_{2}$ is given by ([26], p. 172),

$$
\rho=12 \int_{0}^{1} \int_{0}^{1} C\left(v_{1}, v_{2}\right) d v_{1} d v_{2}-3
$$

The lower and upper bounds of the Kendall's $\tau$ in (b) of Lemma 9 can be obtained based 
on the following relationship between the Kendall's tau and the Spearman's rho [27],

$$
-1 \leq 3 \tau-2 \rho \leq 1
$$


on the following relationship between the Kendall's tau and the Spearman's rho [27],

$$
-1 \leq 3 \tau-2 \rho \leq 1
$$

